\title{
Diagnostic dilemma of hyaline cystic degeneration of uterine fibroids
}

\begin{abstract}
Various forms of degenerations may occur in fibroids but cystic and myxoid degenerations are not common. We reported two case series of cystic degenerations in pregnant and nonpregnant conditions. A myomectomy on pregnant uterus and total abdominal hysterectomy and bilateral salpingoophorectomy was respectively performed successfully. There was diagnostic dilemma in both cases prior to surgical intervention as available imaging investigations made diagnosis of ovarian cystic mass. Magnetic resonant imaging was not available but evidence suggests preoperative use may resolve the diagnostic dilemma.
\end{abstract}

Volume 10 Issue $3-2019$

\author{
Anyanwu M,',2 Gassama K,' Kandeh M' \\ 'Edward Francis Small Teaching Hospital Banjul the Gambia \\ (EFSTH), Gambia \\ ${ }^{2}$ University of the Gambia Medical School, Gambia
}

\begin{abstract}
Correspondence: Matthew Anyanwu, Senior Lecturer University of the Gambia School of Medicine and Allied Sciences department of Obstetrics and Gynaecology, University of the Gambia Medical School, P.O.BoxI646 UTG Banjul, Gambia, Tel (220)-9922933 or (220)-7786700,

Email anyanwum@yahoo.com
\end{abstract}

Received: April 28, 2019 | Published: May 28, 2019

\section{Introduction}

As fibroids enlarge, they outgrow their blood supply which may evoke cascade of inflammatory reactions and ischaemic changes leading to various types of degenerations. Hyaline $(60 \%)$; Cystic (4\%); Red (3\%), Myxoid (1-3\%); Calcified (4\%); Sarcomatous degeneration $(0.1-0.8 \%){ }^{1}$ Hyaline cystic degenerations are rare as only case reports were found in the literature. ${ }^{1}$ The distinctive description of our second case series was a combination of 3 possible degenerations (Hyaline, Cystic and Myxoid) which is extremely rare. The sonographic features were complex; together with the patient's clinical features was indistinguishable from ovarian tumour. They mimic ovarian tumour causing diagnostic dilemma.

\section{Case I}

A 30 year old multipara in her 4th pregnancy at 11 weeks gestation presented with acute abdominal pain and haemodynamic instability. Ultrasound revealed cystic mass, posterior to the gravid uterus of 11 weeks 2 days gestation. A laparotomy was done and a subserous cystic degenerated fibroid at the posterior wall measured $6 \times 7 \mathrm{~cm}$ was resected haemostasis secured.

\section{Case 2}

Mrs BJ a 38 Year old nulliparous lady presented with abdominal pain and swelling for 5 years and irregular painful menses for 3years. She had 2 previous surgeries, a myomectomy 2009 and laparotomy 2013 at the surgical department, nothing was removed. She was cachectic; mildly pale, anicteric and all vital signs were within normal range. Abdomen was distended, midline scar from Xyphysternum to pubic symphysis, measured $41 \mathrm{~cm} \times 38 \mathrm{~cm}$; $S F H$ ? $41 \mathrm{~cm}$; fluid thrill was positive.

Investigations were performed and the results were as follows; HB: 9.9g/dl; Group: "O" Negative 3 pints of blood was crossed matched; FBC/RFT/LFT: were all normal; HCG: NEGATIVE, $\mathrm{HIV}=$ Negative; Alpha Feto protein=negative; LDH=340 [303---618]; HBsAg=Negative; Total BiL: 5.0umol/L; CA 125 Not Available; Chest X-Ray and ECG=normal. Ultrasound and CT -Scan suggested
“Ovarian Malignancy”!! At laparotomy a huge cyst of $41 \mathrm{~cm} \times 38 \mathrm{~cm}$ originating from the anterior uterine wall with multiple adhesions, cystic fluid measured about 8.2 litres. She had uneventful postoperative recovery and was discharged on day 5 . The histopathology result confirmed hyaline cystic degeneration of uterine fibroids. The preoperative imaging mimicked ovarian tumour. A diagnostic dilemma!!

Figure 1 shows the sonographic image of the patient with cystic and solid areas and some characteristics similar to papillary projections (Figure 2) (Figure 3).

Figure 4 shows the sac after drainage of myxoid fluid of 8.2 litres.

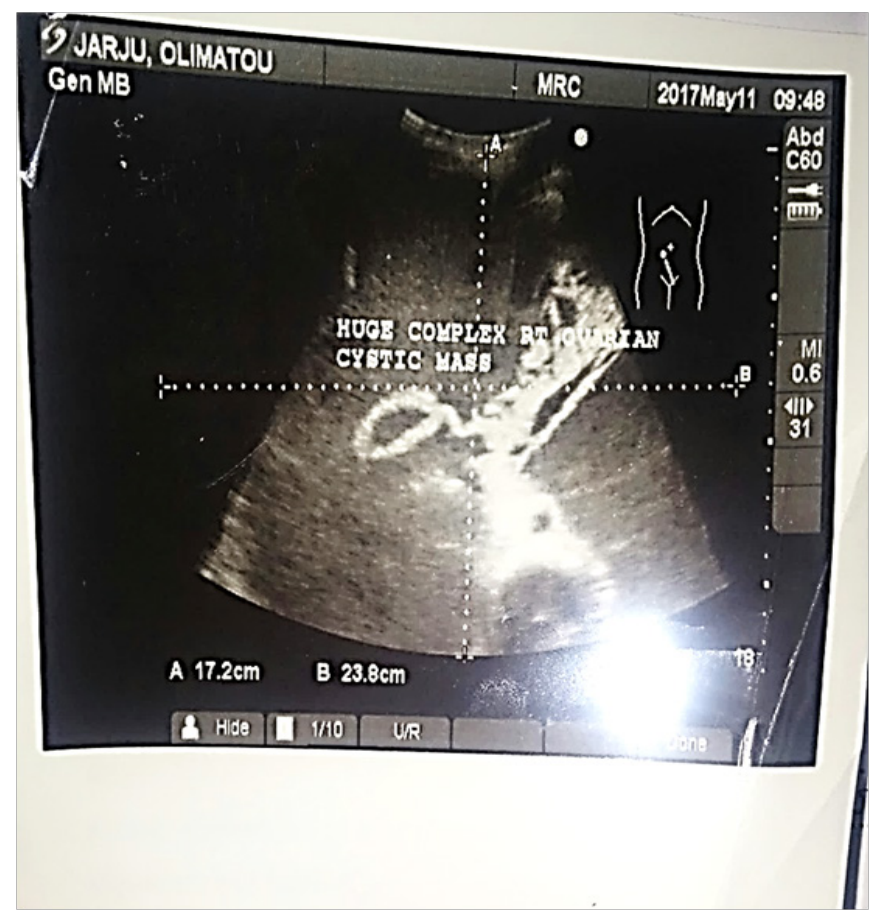

Figure I Published with patient's consent. 


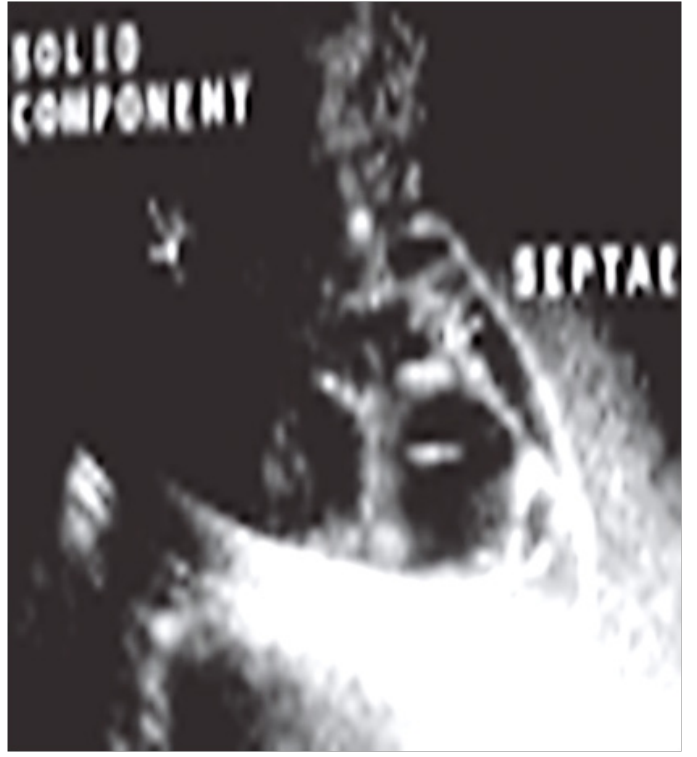

Figure 2 cystic mass not ovarian but uterine?

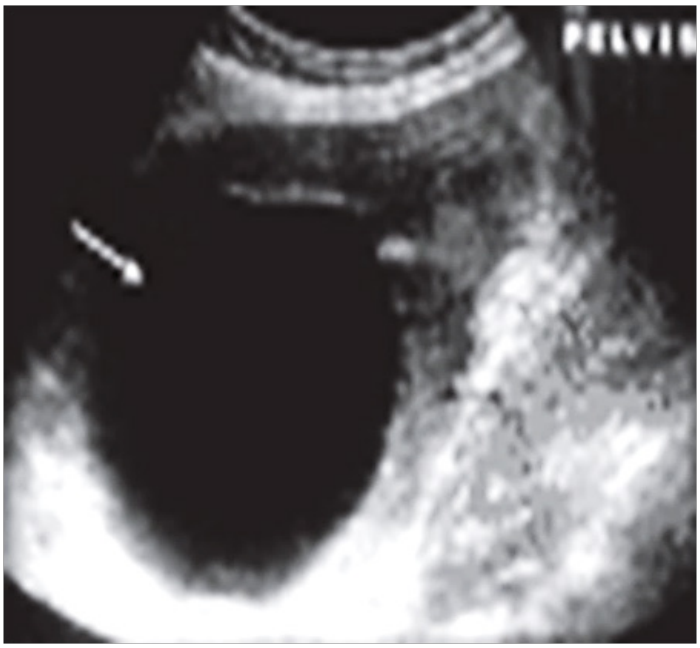

Figure 3 Uterine cystic mass.

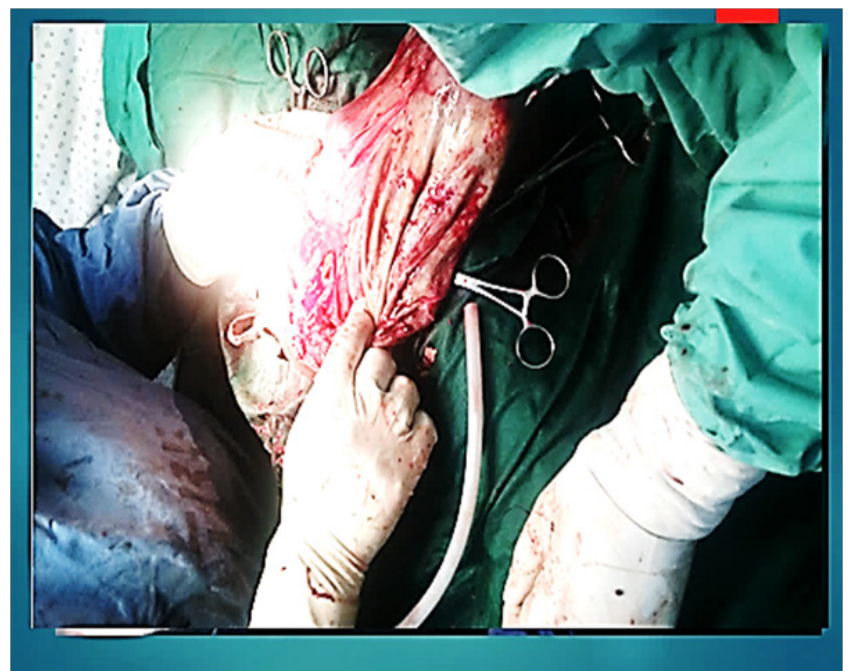

Figure 4 published with consent and permission of the patient.

\section{Discussion}

A leiomyoma or fibroid is a benign tumour presenting as the most common uterine neoplasm with a prevalence of $20 \%-30 \%$ in the women of reproductive age and more than $40 \%$ of women above 40 years of age ${ }^{2}$

As fibroid is common in reproductive age group therefore pregnancy coexisting with fibroid is not uncommon with a prevalence rate estimated at $10.7 \%$ in the first trimester. ${ }^{3}$ Varieties of fibroid degenerations can also occur in pregnancy. Red degeneration of fibroid in pregnancy is a well-established debilitating condition in the second and third trimesters. The basis for antenatal myomectomy stems only from case reports and small case series of up to 18 women. ${ }^{4}$ Therefore, it may not be a routine practice but required appropriate justification such as for severe pain from a degenerating fibroid, a large or rapidly enlarging fibroid, fibroids greater than $5 \mathrm{~cm}$ in diameter within the lower uterine segment, or torsion of a pedunculated fibroid. ${ }^{5-9}$ The most suitable time to undertake a myomectomy is considered to be during the first and second trimesters. ${ }^{5}$ In our case series the management was by antenatal myomectomy due to severe pain which was from the cystic degeneration of a posterior subserous fibroid. The diagnosis was made at surgery. Preoperatively the ultrasound diagnosis was a complex ovarian cyst in pregnancy. As pregnancy was less than 13 weeks and conservative management was not able to improve clinical condition, myomectomy was performed and adequate haemostasis was achieved. Patient made good recovery and pregnancy was carried to term. Caesarean section was performed and a live birth was achieved.

\section{Case 2}

The unavailability of CA 125 also added to the dilemma of arriving at a diagnosis. Although in premenopausal women, elevated CA-125 may occur in benign conditions including fibroids. However, the rate of elevation may not be comparable with malignant epithelial tumours of the ovary. The CT-scan was not able to distinguish the origin of the tumour but was in agreement with the ultrasound findings of a cystic mass with features suggestive of ovarian malignancy. We do not have MRI at the time and could not have done it. However, if MRI was performed probably we could have arrived at a diagnosis before surgery. Similar to case 1 as MRI is safe in pregnancy ${ }^{10}$ we could have made the diagnosis prior to surgery. The study by Kaushik, et al. ${ }^{1}$ managed two cases of cystic degeneration of fibroids. They had diagnostic dilemma and the diagnosis was confirmed at surgery. Tumour resection and hysterectomy was performed. The management was not different from ours as there was no preoperative diagnosis with primary intention to perform primary ovarian malignancy surgery total abdominal hysterectomy and bilateral salpingoophorectomy (TAH-BSO).

In 2004 and 2005 Low SCA et al. ${ }^{2}$; Maria Luisa et al. ${ }^{11}$ respectively published a case of cystic leiomyoma mimicking an ovarian malignancy. The clinical features and investigations mimicked ovarian tumour. The diagnosis was at surgery. They managed by TAH BSO which was similar to how we managed our patient.

However, some radiologists working elsewhere had job satisfaction when their case series of radiological suspected cystic degenerations of the uterine fibroid were confirmed post -surgery when histology report was retrieved Hricak et al. ${ }^{12}$

In 2007; Maizlin et al. ${ }^{13}$ published a paper with this title: "Is It a fibroid? Are you sure?: the sonographic features supported ovarian 
tumour but their worries was resolved by MRI which assisted in the preoperative diagnosis of cystic degenerations of fibroids. The intra-operative findings were in agreement with MRI diagnosis. The availability and application of MRI made the difference in the management of their patient.

The place of MRI in the pre-operative diagnosis cannot be overemphasized. ${ }^{14-16}$ The images below from MRI using T1 T2 weighted views delineate the origin of cystic mass better than other imaging techniques and should be well applied were appropriate in the preoperative management of patient's with massive cystic swelling of the abdomen. The cost implication and inaccessibility of this tool limits the use in routine gynaecological practice (Figure 5) (Figure 6).

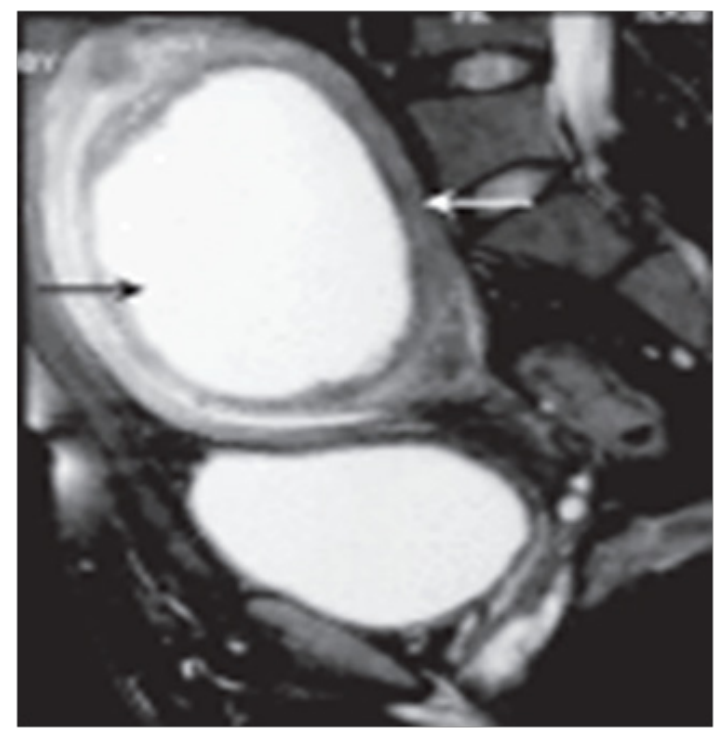

Figure $5 \mathrm{~T} 2 \mathrm{~W}$ published with permission.

T2W

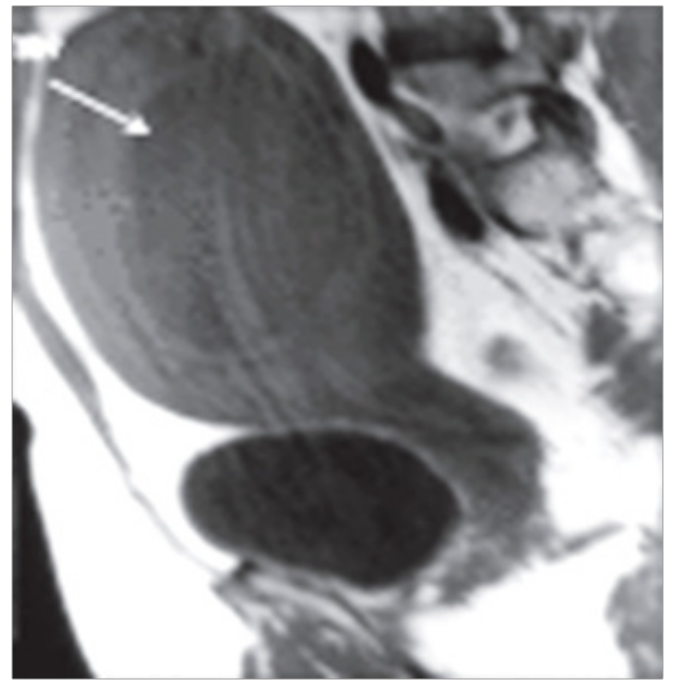

Figure 6 TIW published with permission.

Sagittal TIW

\section{Challenges in the management}

There was insufficient documentation during her initial clinical visit in case 2. No full documentation about the surgery done in 2013 (open \& close surgery?).
Her clinical features and investigations from different places suggest ovarian cancer. CA125 was requested but not available.

Intra operative findings in these case series were different from what we thought and the Surgery was sensitive and difficult in case 1 and 2 respectively. Case 2 was difficult because of the previous surgeries causing massive adhesions and ovaries buried in the uterus which was unavoidably removed in the young age (38 years). Histopathology results confirmed uterine fibroids. A diagnostic dilemma!!!!!

\section{Conclusion}

Hyaline cystic degenerations of the uterine fibroids may be rare but not uncommon. It can occur in pregnancy and non-pregnant state in the reproductive age. Myomectomy in pregnancy is as yet reported in case series. In non - pregnant it may appear so massive with features suggestive of ovarian malignancy. However, with MRI, preoperative diagnosis may be achieved. The distinctive description of our second case series was a combination of three possible degenerations (Hyaline, cystic and Myxoid) which is extremely rare.

\section{Acknowledgments}

None.

\section{Conflicts of interest}

Author has no conflict of interest to declare.

\section{References}

1. Chhavi Kaushik, Akhila Prasad, Yashvant Singh, et al. Case series: cystic degeneration in uterine leiomyomas. Indian $J$ Radiol Imaging. 2008;18(1): 69-72.

2. Low SC, Chong CL. A case of cystic leiomyoma mimicking an ovarian malignancy. Ann Acad Med Singapore. 2004;33(3):371-374.

3. Laughlin SK, Baird DD, Savitz DA, et al. Prevalence of uterine leiomyomas in the first trimester of pregnancy: an ultrasound screening study. Obstet Gynecol. 2009;113(3):630-635.

4. Sampat K, Alleemudder DI. Fibroids in pregnancy: management and outcomes. Obstetrician \& Gynaecologist. 2018;20(3):187-195.

5. Exacoust C, Rosati P. Ultrasound diagnosis of uterine myomas and complications in pregnancy. Obstet Gynecol. 1993;82(1):97-101.

6. De Carolis S, Fatigante G, Ferrazzani S, et al. Uterine myomectomy in pregnant women. Fetal Diagn Ther. 2001;16(2):116-119.

7. Wittich AC, Salminen ER, Yancey MK, et al. Myomectomy during early pregnancy. Mil Med. 2000;165(2):162-164.

8. Febo G, Tessarolo M, Leo L, et al. Surgical management of leiomyomata in pregnancy. Clin Exp Obstet Gynecol. 1997;24(2):76-78.

9. Celik C, Acar A, Cic ek N, et al. Can myomectomy be performed during pregnancy? Gynecol Obstet Invest. 2002;53(2):79-83.

10. Society of pediatric radiology. Fetal MRI - general information. Virginia, USA; 2016.

11. Fogata ML, Jain KA. Degenerating cystic uterine fibroid mimics an ovarian cyst in a pregnant patient. J Ultrasound Med. 2006;25(5):671674.

12. Hricak H, Tscholakoff D, Heinrichs L, et al. Uterine leiomyomas: Correlation of MRI, histopathologic findings and symptoms. Radiology. 1986;158(2):385-391.

13. Maizlin, Zeev V Vos, Patrick M, et al. Is it a fibroid? Are you sure? sonography with MRI assistance. Ultrasound Q. 2007;23(1):55-62. 
14. Karim S Ahamed, Gregory S Raymond. Answer to case of the month \#103 large subserosal uterine leiomyoma with cystic degeneration presenting as an abdominal mass. Can Assoc Radiol J. 2005;56(4):245-247.

15. Okizuka H, Sugimura K, Takemori M, et al. MR detection of degenerating uterine leiomyomas. J Comput Assist Tomogr. 1993;17(5):760-766.
16. Karasick S, Lev-Toaff AS, Michael E Toaff. Imaging of uterine leiomyomas: pictorial essay. Am J Roentgenol. 1992;158(4):799-805. 